\title{
HIGHLIGHTS
}

PROSTATE CANCER

\section{Anterior perirectal fat: a barrier to optimal outcomes in patients undergoing HIFU therapy}

High-intensity focused ultrasound (HIFU) is a relatively new treatment for men with prostate cancer, which is less invasive than the gold standard of prostatectomy. It involves coagulation and ablation of tumor using focused ultrasound beams generated within a rectal probe. While obesity is associated with unfavorable pathologic features and an increased risk of biochemical

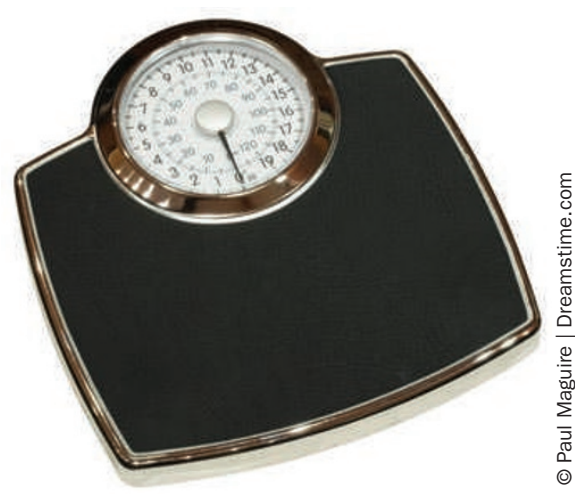

recurrence following radical prostatectomy, the effects of high BMI in men undergoing HIFU are not known. An increased amount of anterior perirectal fat tissue (APFT) between the ultrasound probe and the target prostate tissue might act like a shield, reflecting the ultrasound waves and preventing full coverage of the focal lesion. Sumitomo and colleagues performed a retrospective analysis of data from 115 Japanese patients to determine whether this measure of obesity is associated with decreased disease-free survival following HIFU.

The patients were classified as being obese $(n=48)$, overweight $(n=24)$ or of normal weight $(n=43)$ according to the Western Pacific Regional Office WHO criteria, which takes into account the difference in body shape and build between Westerners and South-East Asians.

The 5-year disease-free survival rates in the obese, overweight and normal-weight groups were 55.5\%, 90.8\% and $83.9 \%$, respectively (log-rank $P<0.01$ ). BMI was significantly correlated with APFT thickness (the mean of thickness measured at three different sites), and multivariate analysis indicated that APFT thickness was a significant predictor of disease-free survival.

The authors conclude that increased APFT thickness could be the cause of poor clinical outcomes following HIFU therapy for prostate cancer. Patient selection on the basis of APFT thickness-for which BMI seems to be a useful surrogate-may lead to improved results in men receiving this treatment.

Nick Warde

Original article Sumitomo, M. et al. Anterior perirectal fat tissue thickness is a strong predictor of recurrence after high-intensity focused ultrasound for prostate cancer. Int. J. Urol. 17, 776-783 (2010) 